\title{
Airline Planning Benchmark Problems Part II: Passenger Groups, Utility and Demand Allocation
}

\author{
Kerem Akartunal1 ${ }^{\mathrm{a}}$, Natashia Boland ${ }^{\mathrm{b}}$, Ian Evans $^{\mathrm{c}}$, Mark Wallace $^{\mathrm{d}}$, Hamish Waterer ${ }^{\mathrm{b}, \mathrm{d}, *}$ \\ ${ }^{a}$ Department of Mathematics and Statistics, University of Melbourne, Parkville VIC 3010, Australia \\ ${ }^{b}$ School of Mathematical and Physical Sciences, University of Newcastle, Callaghan NSW 2308, Australia \\ ${ }^{c}$ Constraint Technologies International, Level 7224 Queen St, Melbourne VIC 3000, Australia \\ ${ }^{d}$ Faculty of Information Technology, Monash University, Caulfield VIC 3145, Australia
}

\begin{abstract}
This paper is the second of two papers entitled "Airline Planning Benchmark Problems", aimed at developing benchmark data that can be used to stimulate innovation in airline planning, in particular, in flight schedule design and fleet assignment. The former has, to date, been under-represented in the optimization literature, due in part to the difficulty of obtaining data that adequately reflects passenger choice, and hence schedule revenue. Revenue models in airline planning optimization only roughly approximate the passenger decision process. However there is a growing body of literature giving empirical insights into airline passenger choice. Here we propose a new paradigm for passenger modelling, that enriches our representation of passenger revenue, in a form designed to be useful for optimization. We divide the market demand into market segments, or passenger groups, according to characteristics that differentiate behaviour in terms of airline product selection. Each passenger group has an origin, destination, size (number of passengers), departure time window, and departure time utility curve, indicating willingness to pay for departure in time sub-windows. Taking as input market demand for each origin-destination pair, we describe a process by which we construct realistic passenger group data, based on analysis of empirical airline data collected by our industry partner. We give the results of that analysis, and describe 33 benchmark instances produced.
\end{abstract}

Keywords: Airline planning, passenger choice models, revenue models, benchmark data

\section{Introduction}

This paper is the second of two papers entitled "Airline Planning Benchmark Problems". Our primary goal in these papers is to stimulate and facilitate further research in airline planning. Currently, real data is difficult to obtain, creating a barrier to entry for many researchers, and limiting the ability of the research community to compare approaches. Each research group or paper typically consider a specific problem variant, with problem specification and data not likely to be available to the broader community. There is also relatively little work that has tackled the first stage of the airline planning process: flight schedule design.

In these two papers, we take some first steps towards addressing these issues, by developing a data generation methodology, and realistic benchmark instances, that provide standardized data with which to initiate the airline planning process. In particular, we focus on the the development of airline demand data, which is critical to schedule design.

Optimization has been key to airline planning for many decades

\footnotetext{
${ }^{*}$ Corresponding author

Email addresses: k. akartunali@ms .unimelb.edu.au (Kerem Akartunal1), natashia.boland@newcastle.edu.au (Natashia Boland), ian.evans@contecint.com.au (Ian Evans), mark.wallace@infotech.monash.edu.au (Mark Wallace), hamish.waterer@newcastle.edu.au (Hamish Waterer)
}

(see Klabjan (2005) or Bazargan (2004), for example). However, as noted in Klabjan (2005), for the most part flight schedule planning is a manual process. Notable exceptions are discussed in two papers, Yan and Tseng (2002) and Yan, Tang, and Lee (2007), on flight scheduling in Taiwan, and in Lohatepanont and Barnhart (2004). Yan and Tseng (2002) formulate schedule design as a kind of network design problem in a time-space network, in which the passengers are represented as explicit multicommodity flows in the network designed by the aircraft variables. There is a commodity for each OD pair, and the total commodity flow is bounded above by the market demand. They use a standard ticket price for each commodity on each flight leg, and apply a "holding cost" to time passengers spend on the ground at intermediate stops, dependent only on OD pair and time spent. The latter is the only way in which passenger utility with respect to alternate itineraries is considered. Yan et al. (2007) extend this work to embed a more sophisticated passenger choice model, in which the presence of competition in the market is modelled by assuming passengers will be unavailable to the airline if they have to wait too long to depart. The passenger loss parameters require a complicated calculation taking into account airline preferences, ticket prices, flight frequency and travel time, which is nonlinear in the decision variables, and seems to be decoupled from subsequent flights in a multi-flight itinerary (only loss at the port of origin is considered). Lohatepanont and Barnhart (2004) combine fleet as- 
signment with incremental flight schedule design, and take into account passenger choice via a spill-and-recapture model based on earlier work of Kniker (1998). Each possible itinerary in a market receives a rating, based on an industry standard known as the Qantitative Share Index $(Q S I)^{1}$, for measuring the "attractiveness" of an itinerary, taking into account time of day of departure, length of trip, and number of connections. This is converted into a probability that passengers will be recaptured by an itinerary if spilled from the preferred itinerary for the market. The measure is independent of fare category, and depends only on market. We note that itinerary-based market share indicators are still a subject of current research, see Coldren, Koppelman, Kasturirangan, and Mukherjee (2003), for example. However like the QSI, Coldren et al. (2003), treat the market (OD pair) as whole, e.g., only average fares for an airline are considered.

A common feature of passenger choice models in schedule design optimization is that passengers with the same OD pair are treated identically. Furthermore the effects of passenger choice on revenue are modelled in a relatively simple way: (i) revenue penalty for long connections in multi-flight itineraries (Yan and Tseng (2002)), (ii) lost passengers due to long waits for departure (Yan et al. (2007)) and (iii) lost passengers at itinerary-based rates with recapture options (Lohatepanont and Barnhart (2004)). However both common sense and emerging research indicate that the passenger market is segmented, with different groups of passengers with the same OD pair showing different passenger choice behaviours, and exhibiting different utility functions with respect to a range of factors. For example, Walker (2006) analyse empirical data to show that passengers with different time-of-day preferences show different utility functions with respect to schedule delay. Walker (2006) also indicates that whether the traveller is business or leisure, or whether the traveller is more sensitive to their arrival time rather than departure time, can affect their utility functions for schedule delay. Koppelman, Coldren, and Parker (2008) also finds differences in schedule delay utility for business versus leisure travellers, and furthermore observes differences depending on whether the trip is out-bound or in-bound. Walker (2006) finds that the business and leisure segments put different dollar figures on factors such as total time for the trip, number of stops, aircraft changes, and so on. This is confirmed by the work of Garrow, Jones, and Parker (2007), who also showed that departure versus arrival sensitivity can affect preferred travel time, with departure-sensitive travellers showing strong morning and evening peaks, with arrival-sensitive passengers having a midday peak.

We conclude that the airline passenger market is segmented, and propose that schedule design optimization should be based on revenue models that better reflect this market segmentation. In particular, we propose to represent airline demand by passenger groups, according to characteristics that differentiate behaviour in terms of airline product selection. We argue that this can be naturally modelled in an optimization setting. For

\footnotetext{
${ }^{1}$ Also sometimes referred to as the Quality Service Index.
}

example, in multi-commodity flow models, such as those of Yan and Tseng (2002) and Yan et al. (2007), a commodity for each passenger group could be defined instead of for each ODpair. In itinerary-based models such as those of Lohatepanont and Barnhart (2004), an extra subscript would be required for passenger-itinerary variables. This is likely to increase model size. However we propose that departure time window should be a defining characteristic of a passenger group, so the subnetwork for each group will be necessarily limited. This will to some extent mitigate increase in model size. Furthermore, dealing with larger models is a challenge the optimization research community tends to enjoy, so we hope this proposal, with its associated benefits for improved revenue modelling, will stimulate such research. Indeed, we have made a small start on such work: our companion paper Akartunalı, Boland, Evans, Wallace, Waterer, and Smith (2009b) studies an integrated airline schedule design and fleet assignment problem, for which passenger groups provide the necessary detailed information.

Clearly substantial new empirical research is required to identify the "best" way of representing passenger groups. In this paper, we have taken a pragmatic approach, applying a mix of common sense and the insights from the empirical literature, together with the insights of our industry partner and their analysis of airline passenger data (Evans (2009)). We view this as a first step, that can be used to test the concept. Thus we focus on what we believe are the key features. Of course a passenger group must have an OD-pair, and represent a specified number of passengers. The business versus leisure divide features in most recent analysis; here we do not explicitly define those as characteristics, but instead associate a fare with each passenger group, representing what they are willing to pay to travel at their preferred time. Since preferred time of day has been identified as having an important impact on schedule delay utility, (e.g. by Walker (2006)), we also characterize passenger groups by their departure time window, and a travel time utility curve, which subtracts schedule delay disutility from the fare the passenger is willing to pay to travel at their preferred time.

In what follows, we describe in detail the methodology we used to construct passenger group data for our benchmark problems, taking as input a flight network, and OD-pair demand, together with other design parameters and template data that we discuss in detail as required. Unlike Part I of this paper, Akartunal1, Boland, Evans, Wallace, and Waterer (2009a), in which an optimization model was developed to solve the inverse problem of deriving OD-demand from observed passenger loads on flight network arcs, the methodology here does not require sophisticated mathematics. However it does require some analysis and modelling of airline data, and careful explanation, so that the resulting benchmarks can be properly understood.

We first, in Section 2, discuss our key assumptions, and define necessary terminology and notation. We also give our template for travel time utility curves. In Section 3, we explain how we determine the number of passenger groups to create for each OD-pair, and how we define values for the time of day, fare and utility curve for each group. In Section 4 we describe how we partition the given OD-pair demand between passenger groups. Our benchmark instances are described in Section 5. 


\section{Assumptions, notation and utility curve templates}

This analysis is restricted to short-haul air travel where most flying occurs during the day and evening, and there is similar flying from one day to the next. In this context, our industry partner observes that most time-sensitive passengers fall into one of three types:

- Those wishing to travel early in the morning, e.g. business travellers who are to work at the destination for a day and return in the evening;

- Those wishing to travel early in the evening, e.g. business travellers returning from a day trip or business travellers travelling the night before to be ready for an early morning meeting; and

- Those preferring to travel at a time that does not involve early rising or late dining, and thus with a preference to travel around the middle of the day.

This categorization is consistent with Table 6.6b in UK Department of Transport (2006), which shows morning and afternoon demand peaks for the purpose of "Business" but a single broad spread centred on the middle of the day for the purpose of "Holiday/Day trip/Other". As time constraints are tighter for the first two types, such passengers are willing to pay a higher fare to travel at their preferred time compared to the third type (noted also by Garrow et al. (2007)).

We conjecture a fourth type of budget-conscious passenger: those who are unconcerned with the time of flying (timeinsensitive), as long as the fare is low. The presence of this type of passenger is inferred from the revenue management strategies that are in place at most airlines around the world. In doing so, we note the difficulty of inferring passenger preferences from flight data. For economic reasons, airlines must keep their high capital-cost assets working continuously, and so must fly during the middle of day, when passenger survey data suggests demand is low. The airlines handle this via revenue management, seeking to attract budget-conscious time-insensitive passengers onto flights in the middle of the day, and by attempting to locate middle-of-the-day capacity on flights between port pairs for which there is low frequency of service, so passengers have little choice.

We refer to these four types of passenger as morning, evening, midday and time-insensitive, respectively.

In all cases, for the sake of simplicity, we assume passengers are departure-time sensitive. Garrow et al. (2007) finds that this is true for the majority of passengers, and also finds that the minority arrival-time-sensitive passengers are generally midday travellers, speculating that hotel check-in times are the cause. In the context of short-haul operations and midday travel, when time zone impacts are relatively minor, we believe preferred arrival time can reasonably be "mapped back" to preferred departure time, and so restricting our attention to departure-time sensitive groups is a reasonable approximation (see also Evans (2009)).

For each of the four passenger types, we propose a different shape of travel time utility curve. The shapes for the first three

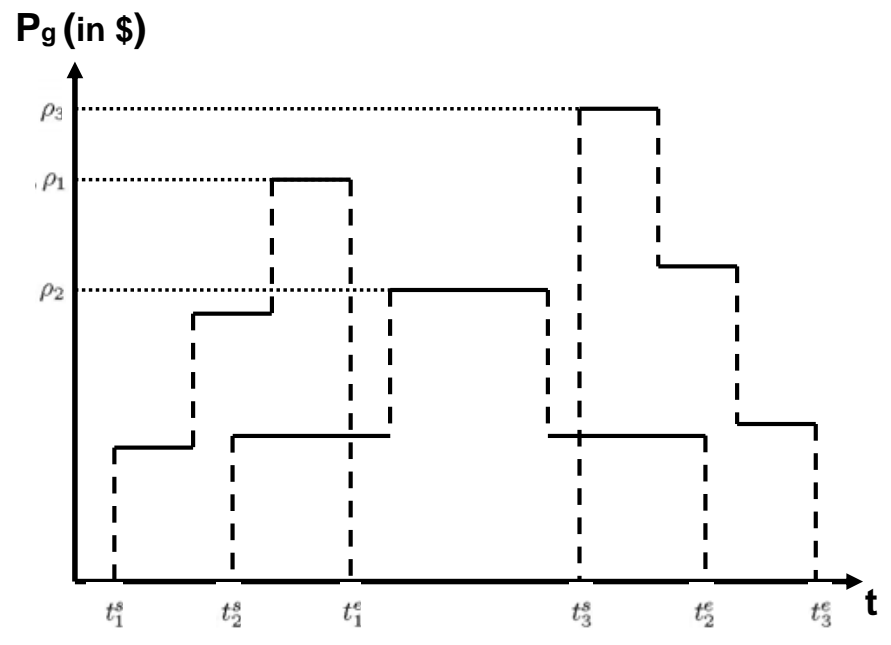

Figure 1: Travel time utility curves for morning, afternoon and evening passenger groups

are shown in Figure 1. Moving from left to right across the time axis, labelled $\mathbf{t}$, we see the morning, midday and evening curves respectively. In all cases, we use a piecewise constant function with three pieces, defined over a time window. For morning passengers, since we assume these are travelling for some scheduled activity at their destination, we assume their preference is to travel at the right time to make the activity, but could be willing to travel earlier if the price was right. For evening passengers, we assume they need to complete some activity at their origin prior to travel (e.g. completing the activity they travelled for before returning home, or completing a day at their home office before travelling for an early start the following day), and so reverse the shape of the curve for morning passengers. Midday passengers simply have a preferred time of travel, and are willing to travel either earlier or later, for the right price. The curve used for time-insensitive passengers is simply a flat line, indicating that they will only fly if the price is right, and then would be willing to fly at any time for that price.

Before discussing our assumptions about these curves further, we first define some notation and terminology. Since we use a daily setting, we let $\mathcal{T}$ denote the length of a day in time units e.g. minutes. So time parameters for passenger groups are chosen from the interval $[0, \mathcal{T}] . G$ denotes the index set of all passenger groups, each $g \in G$ having an origin $o_{g}$ and destination $d_{g}$. There are $\eta_{g}$ passengers in group $g \in G$. Each group $g \in G$ also has an earliest departure time, $t_{g}^{s}$, and latest departure time, $t_{g}^{e}$, defining the start and end of the time window $\left[t_{g}^{s}, t_{g}^{e}\right]$ in which the passengers from this group are willing to travel. Each group $g \in G$ has a preferred interval for travel, $\left[\bar{t}_{g}^{s}, \bar{t}_{g}^{e}\right] \subseteq\left[t_{g}^{s}, t_{g}^{e}\right]$, and a fare they are willing to pay to travel in that interval, $\rho_{g}$. We call these the peak interval and peak fare respectively; these correspond to the peaks in the utility curves shown in Figure 1.

Given the time window, peak interval and peak fare for a passenger group, the rest of the utility curve is specified by a fixed formula, for each passenger type. Based on independent studies (see, e.g. Commission of the European Communities (2007), Civil Aviation Authority (2008)), we assume morning 
and evening passenger groups consist of about $25 \%$ business travellers and $75 \%$ leisure travellers, and that the proportion of business travellers in any midday group is negligible. We take the value of time to be $v^{B}=\$ 68.97$ and $v^{L}=\$ 19.64$ per hour for business and leisure travellers respectively, based on the study of Garrow et al. (2007), and thus the value of time for morning and evening groups to be on average $v=0.25 v^{B}+0.75 v^{L}$, and $v^{L}$ for midday groups. We assume the utility curves have identical width $u_{g}=\bar{t}_{g}^{e}-\bar{t}_{g}^{s}$ for each of the three pieces, and so can compute the formulae

$$
P_{g}(t)= \begin{cases}\rho_{g}, & \bar{t}_{g}^{s} \leq t \leq \bar{t}_{g}^{e} \\ \rho_{g}-v u_{g}, & \bar{t}_{g}^{s}-u_{g} \leq t \leq \bar{t}_{g}^{s} \\ \rho_{g}-2 v u_{g}, & \bar{t}_{g}^{s}-2 u_{g} \leq t \leq \bar{t}_{g}^{s}-u_{g}\end{cases}
$$

for morning groups $g$, a similar formulae for evening groups, while for midday groups $g$ the formula is simply

$$
P_{g}(t)= \begin{cases}\rho_{g}, & \bar{t}_{g}^{s} \leq t \leq \bar{t}_{g}^{e} \\ \rho_{g}-V^{L} u_{g}, & t \in\left[\bar{t}_{g}^{s}-u_{g}, \bar{t}_{g}^{s}\right] \cup\left[\bar{t}_{g}^{e}, \bar{t}_{g}^{e}+u_{g}\right] .\end{cases}
$$

Of course time-insensitive passengers simply have utility function $P_{g}(t)=\rho_{g}$ for all $t \in[0, \mathcal{T}]$.

Other key assumptions we make in generating passenger group data for our benchmark instances are as follows. If the user has additional data to hand, it would not be hard to relax these assumptions and still apply the methodology.

- We assume that each OD-pair has a single time-insensitive passenger group, and that the proportion of time-insensitive passengers for each OD pair is constant, i.e. there is a single constant, which we refer to as $\phi_{\text {tm_ins }} \in[0,1]$, so that the proportion of passengers in an OD market allocated to the time-insensitive group is $\phi_{\text {tm_ins }}$, irrespective of OD pair.

- We assume that for any given OD pair, there is no overlap between peak intervals for the passenger groups with that OD pair (other than with the time-insensitive group, which has a single "peak" interval covering the whole time period). In effect, the peak interval (preferred time of travel) for morning, midday and evening groups uniquely defines the group. Ideally, we would have alternative passenger groups, with different utilities, sharing a preferred travel time. However at present there is no data to support this level of detail.

- For each OD pair $(o, d)$, there is a basic length of time, which we call the unit width for that pair, denoted by $\mu_{o, d}$, so that for all passenger groups $g \in G$ with $o_{g}=o$ and $d_{g}=d, u_{g}=\mu_{o, d}$ for $g$ a morning or evening group, and $u_{g}=2 \mu_{o, d}$ for $g$ a midday group. The difference for midday travellers is due to our assumption that their preferred travel time arises from a desire to avoid early or late travel, rather than from a need to travel before or after a particular activity, and our assumption that they are predominantly leisure travellers. Both factors imply greater flexibility for midday groups. The use of a unit width for each OD pair reflects the idea that passengers' willingness to be flexible in the time they travel is a function of the frequency of service on the OD-pair; clearly passengers in remote locations expect to wait longer for service, whereas passengers travelling between major cities will expect a flight close to their preferred travel time. In other words, the more itineraries available passengers from an OD pair, the narrower their utility curve will be.

We discuss the latter point further in the next section.

\section{Creating passenger groups}

In this section, we describe how passenger groups are created, and how the time of day data, as well as the data defining the travel time utility curves, are determined for each group.

Calculating unit width. In order to generate specific passenger groups and data values for those groups, we first calculate the unit width value $\mu_{o, d}$ for each OD pair $(o, d)$. As mentioned above, we postulate that passengers willingness to be flexible about when they travel, (and hence the unit width), depends on the frequency of service they expect to see on that OD pair. At first sight, one might think this is a function of $D_{o, d}$, the given market demand for OD pair $(o, d)$ (total number of passengers wishing to travel (daily) from $o$ to $d$, generated as described in Akartunali et al. (2009a)). However this figure could be misleading. To illustrate, consider an OD pair $(o, d)$ where the flight network includes direct flights, but where there is also a reasonable one-stop service via port $i$. Then the frequency of the one-stop service depends on the expected frequency for OD pairs $(o, i)$ and $(i, d)$. This could get quite difficult to estimate, since these in turn depend on expected frequency on indirect paths, so for simplicity, we assume that frequency of one-stop services (or other indirect services), such as $(o, i, d)$, can be well estimated from the OD demands $D_{o, i}$ and $D_{i, d}$. We assume whichever is the smaller of these creates a bottleneck, and the frequency of service is predicated on that value. We thus solve a maximum flow problem for each OD-pair, to maximize the flow from $o$ to $d$ on all reasonable paths from $o$ to $d$ in the flight network (our definition of "reasonable" is given in Akartunalı et al. (2009a)), with arc capacities give by the values $D_{i, j}$ for each $\operatorname{arc}(i, j)$. We call the resulting maximum flow value the surrogate demand, denoted by $\bar{D}_{o, d}$ for each OD pair $(o, d)$. This is converted to approximate the number of services that might be available to the OD pair, $N_{o, d}^{s e r v}$, by using the average aircraft capacity, while accounting for average load factors. We also scale down by $1-\phi_{\text {tm_ins }}$, since airlines typically do not put on services to meet the low-budget demand, instead using revenue management to manipulate that demand to "fill gaps".

To determine unit width $\mu_{o, d}$ from expected service frequency $N_{o, d}^{s e r v}$, we assume that passengers are willing to be flexible enough to wait for the interval of time between services, without reduction in their utility, and simply assume an even spread of service over the available time. Since most airports do not operate 24 hours per day, we define the airport operating interval, which we take to be 18 hours, (e.g. if the port operates from 5 am to 
$11 \mathrm{pm})$, denoted by $\mathcal{T}_{\text {oper }}$, and assume services are spread across this interval. Thus unit width can calculated simply as

$$
\mu_{o d}=\mathcal{T}_{\text {oper }} / N_{o, d}^{\text {serv }}
$$

Creating passenger groups. Since unit width determines the width of the peak interval, $u_{g}$, for each passenger group $g$, and since we make the assumption that no two passenger groups (other than the time-insensitive group) can have overlapping peak intervals, we see that creating the passenger groups for each OD pair is simply a matter of stepping through time. (We also assume that each time is in a peak interval for some passenger group. If the demand profile doesn't support this, then we would expect the passenger group to be assigned zero passengers in Section 4, so the group could be ignored.) Defining morning to end at time $T_{m}$ and evening to start at time $T_{e}$, we can simply create a passenger group for each interval of length $\mu_{o, d}$ from the start of the day until $T_{m}$, then create a group for each interval of length $2 \mu_{o, d}$ until $T_{e}$, and finish by creating a group for each interval of length $\mu_{o, d}$ until the end of the day. (In addition, we must create the single time-insensitive group: this is explained in Section 2.) However depending on how nicely $\mathcal{T}, T_{m}$ and $T_{e}-T_{m}$ divide by $\mu_{o, d}$, this could lead to a somewhat skewed collection of passenger groups. So instead we start at the centre of the day, and work outwards. Our algorithm is specified as Algorithm 1. It is helpful to note that if the peak interval $\left[\bar{t}_{g}^{s}, \bar{t}_{g}^{e}\right]$ falls so that its mid-point is at time $T_{m}$ or earlier, it is deemed a morning group, at time $T_{e}$ or later an evening group, and otherwise it is a midday group. The first while loop calculates the time characteristics for intervals before midday, taken to be $\left(T_{m}+T_{e}\right) / 2$, and the second while loop does it for after midday intervals. We use $A$ to denote the set of morning ("am") groups, $M$ to denote the set of midday groups, and $P$ to denote the evening ("pm") groups created for an OD pair. Note that we only explicitly describe calculation of the peak interval parameters $\bar{t}_{g}^{s}$ and $\bar{t}_{g}^{e}$ for each group $g$, since all other times, including $u_{g}$, can be calculated from these or from $\mu_{o, d}$ using the formulae given in Section 2 and the knowledge of which type of group it is.

As Algorithm 1 is stated, it creates passenger groups across the whole time interval $[0, \mathcal{T}]$. However, as mentioned earlier, some airports may have constrained operating hours, and this may affect passenger expectations for travel. In such cases, one could just take zero to be first time at which passengers may be willing to travel, and replace $\mathcal{T}$ in Algorithm 1 with the length of the time interval over which passengers wish to travel.

We note that this algorithm and some of our assumptions might need to be adjusted for longer flights, particularly in a west-to-east direction, when changes in time zones would seem likely to affect passenger preferences. We discuss this point further in Section 4.

To complete our specification of the travel time utility curve for each passenger group, we need to determine the peak fare $\rho_{g}$ for each group $g$.

Calculating peak fares. For each passenger group, we need to determine the fare passengers in the group are willing to pay to
Data: Unit width $\mu_{o, d}$ for each OD pair $(o, d)$

Result: The set of passenger groups $G$, together with time characteristics of each group $g \in G$

Initialize $g:=0$;

forall $O D$ pairs $(o, d)$ do

Set $A:=\emptyset, M:=\emptyset$ and $P:=\emptyset$;

Set $g:=g+1, o_{g}:=o$ and $d_{g}:=d$;

Create the time-insensitive group:

set $\bar{t}_{g}^{s}:=0$ and $\bar{t}_{g}^{e}:=\mathcal{T}$;

if $\mu_{o d} \leq \frac{\mathcal{T}}{2}$ then

Set $g:=g+1, o_{g}:=o$ and $d_{g}:=d$;

Create a first midday group:

calculate $t:=\frac{T_{m}+T_{e}}{2}$;

set $\bar{t}_{g}^{s}:=t-\mu_{o d}$ and $\bar{t}_{g}^{e}:=t+\mu_{o d}$;

add $g$ to $M$;

Set $t:=t-\mu_{o d}$;

while $t>0$ do

Set $g:=g+1, o_{g}:=o$ and $d_{g}:=d$;

if $t-\mu_{o d} \geq T_{m}$ then

Create another midday group: set $\bar{t}_{g}^{s}:=t-2 \mu_{o d}$ and $\bar{t}_{g}^{e}:=t$; add $g$ to $M$;

Set $t:=t-2 \mu_{o d}$;

else

Create a morning group:

set $\bar{t}_{g}^{s}:=t-\mu_{o d}$ and $\bar{t}_{g}^{e}:=t$;

add $g$ to $A$;

Set $t:=t-\mu_{o d}$;

end

end

Set $t:=\frac{T_{m}+T_{e}}{2}+\mu_{o d}$;

while $t<\mathcal{T}$ do

Set $g:=g+1, o_{g}:=o$ and $d_{g}:=d$;

if $t+\mu_{o d} \leq T_{e}$ then

Create another midday group: set $\bar{t}_{g}^{s}:=t$ and $\bar{t}_{g}^{e}:=t+2 \mu_{o d}$; add $g$ to $M$;

Set $t:=t+2 \mu_{o d}$;

else

Create an evening group: set $\bar{t}_{g}^{s}:=t$ and $\bar{t}_{g}^{e}:=t+\mu_{o d}$; add $g$ to $P$;

Set $t:=t+\mu_{o d}$

end

end

end

end

Set $G:=\{1, \ldots, g\}$

Algorithm 1: Creating passenger groups and their time characteristics 
fly at their preferred time. To do this, we adopt a standardized fare profile, which describes fare variation by time of day, irrespective of OD pair. The fare profile is thus a function $f(t)$ defined over $t \in[0, \mathcal{T}]$. We discuss this function and how we arrive at it in more detail below. For now, we note only that it is normalized, and applied to a specific OD-pair by scaling with OD-specific costs, for example, related to the shortest path in the flight network between the origin and destination. This gives a fare profile, $f_{o, d}(t)=\alpha_{o, d} f(t)$, specific to OD pair $(o, d)$, where $\alpha_{o, d}$ is the scale factor. For each passenger group $g \in G$, we then calculate the average fare over its peak interval to arrive at the peak fare, i.e. we set

$$
\rho_{g}=\frac{\int_{t=\bar{t}_{g}^{s}}^{\bar{T}_{g}^{e}} f_{o_{g}, d_{g}}(t) d t}{\bar{t}_{g}^{e}-\bar{t}_{g}^{s}} .
$$

The scale factor $\alpha_{o, d}$ is computed so that the minimum fare seen in $f$ maps to an estimate of the lowest fare one would expect to see for travel from $o$ to $d$. We take this to be $b+t_{o, d}^{\min } r$, where $b$ and $r$ are assumed to be industry standard values indicating the fixed cost and per unit flight time charge, per passenger, per trip respectively, and $t_{o, d}^{\mathrm{min}}$ indicates the least flight time a passenger could reasonably expect to accumulate in travelling from $o$ to $d$ in the given flight network. For these first two parameters, we use the current industry standards of $b=\$ 50.50$ and $r=\$ 0.60$ per minute flight time. Thus $\alpha_{o, d}$ can be calculated by

$$
\alpha_{o, d}=\left(b+t_{o, d}^{\min } r\right) / \min _{t \in[0, \mathcal{T}]} f(t) .
$$

We note that for each time-insensitive group $g$, we simply set $\rho_{g}$ to be the minimum fare that could be expected for its OD pair, i.e. $\rho_{g}=b+t_{o_{g}, d_{g}}^{\min } r$.

To determine peak fares for time-sensitive groups, we now return to discuss our standardized fare profile, $f(t)$, in more detail. To derive such a profile, our industry partner analysed fare data on two typical busy mid-week days on a single $700 \mathrm{~km}$ short-haul route between two large cities with a high number of flights. This could be classed as a "shuttle" route, i.e. a relatively short route with a high frequency of service. The reason for analysing such a route is the hope that it would reveal willingness to pay, independent of offered capacity, and independent of strategies used by airlines to smooth demand. Such a route would also maximize the length of time over which data points could be observed.

The airline chosen for analysis was one with the the following characteristics: (i) on the route selected, the interval between flights ranged from 15 minutes to one hour according to the expected passenger load, and (ii) its revenue management system includes multiple fare categories, with higher fares at times where there is more demand and where customers have proven willing to pay higher prices to travel at those times. These characteristics enabled us to gather fare data from the airline's web booking system. Data comprising the lowest available fare and the number of scheduled flights in each hour was averaged over flights in both directions on the route to minimise the effects of "waves" of flights (sometimes known as "bank-

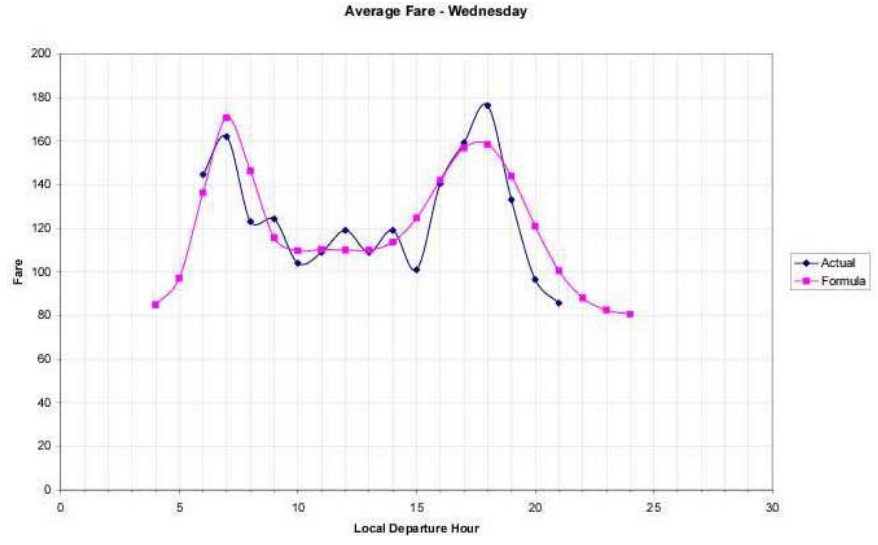

Figure 2: Actual and fitted fare profiles

\begin{tabular}{cccc}
\hline Type $(y)$ & $\tau_{y}$ & $\sigma_{y}$ & $w_{y}$ \\
\hline Morning & 7.0 & 1.0 & 75 \\
Midday & 11.0 & 3.5 & 30 \\
Evening & 17.75 & 2.0 & 75 \\
\hline
\end{tabular}

Table 1: Fare profile function parameters

ing"), and compared between two days to give an indication of variability.

Both days showed morning and evening peaks at the same time (around 7am and 6pm respectively, which appear to be very close to the peaks in Figures 2 and 4 of Koppelman et al. (2008)). Both also showed soft midday peaks. However there was significant variation between the two days in terms of relative fare values: Tuesday's morning peak was much higher than its evening peak, whereas the two peaks for Wednesday (see Figure 2), were of similar height (see Evans (2009) for further details).

The data for these two days was fitted with a combination of 3 normal distribution functions, one for each of the morning, midday and evening peaks, i.e. we constructed

$$
f(t)=h^{a}(t)+h^{m}(t)+h^{e}(t)
$$

where each function took the form

$$
h^{y}(t)=w_{y} \exp \left(-\frac{\left(t-\tau_{y}\right)^{2}}{2 \sigma_{y}^{2}}\right)+80
$$

for each $y \in\{a, m, e\}$, where $\tau_{y}$ and $\sigma_{y}$ are the two normal distribution function parameters (representing mean and standard deviation respectively), and $w_{y}$ is a weighting factor. ( $\$ 80$ was the "baseline" fare for this route.) The parameters found to best fit the two days of data are shown in Table 1, and resulting function $f$ is shown in Figure 2.

Like the passenger group peak interval definitions, the fare profile might need to be adjusted for longer flights, particularly in a west-to-east direction. We discuss this further in the next section. 


\section{Demand allocation}

The final step of our approach is to partition market demand $D_{o, g}$ for each OD pair, $(o, g)$, (calculated in our first paper Akartunali et al. (2009a)), amongst the passenger groups for that OD pair. We call this step demand allocation.

For each time-insensitive group $g$ the number of passengers associated with this group, $\eta_{g}$, is simply

$$
\eta_{g}=\phi_{\text {tmins }} D_{o_{g}, d_{g}}
$$

where $\phi_{\text {tm_ins }}$ is the fixed proportion of time-insensitive passengers, unless no other passenger groups were generated for this OD pair. The latter case could occur if the surrogate demand for the OD-pair is very low, in which case $\mu_{o, d}$ exceeds $\mathcal{T} / 2$. If this occurs, the time-insensitive group is the only group for its OD pair, and so we set $\eta_{g}$ to the entire market demand:

$$
\eta_{g}=D_{o_{g}, d_{g}}
$$

As for the peak fare calculation, for time-sensitive groups we use a standardized demand profile: a function $\mathbf{d}(t)$ for each $t \in[0, \mathcal{T}]$ that indicates the proportion of demand seen at time $t$. We discuss below how we arrive at this function. The function is normalized so that the area under the curve is 1 , and then the number of passengers $\eta_{g}$ associated with passenger group $g \in G$ is calculated as follows:

$$
\eta_{g}=\left(1-\phi_{\text {tm_ins }}\right) D_{o_{g}, d_{g}}\left(\int_{t=\bar{t}_{g}^{s}}^{\bar{T}_{g}^{e}} \mathbf{d}(t) d t\right) .
$$

We note that for both the fare and demand profiles, our main motivation for using standardized profiles is the lack of adequate data to support alternatives. Whilst passenger load data is generally more readily available than fare data, both types of data can only represent preferences for the offered itineraries, which for many OD pairs can be very infrequent. By contrast data derived from passenger surveys would be ideal for our purposes. However the literature in this area is quite sparse, (e.g. Walker (2006), Garrow et al. (2007)), and the data collected to date is not rich enough to allow us to differentiate demand profiles by OD pair characteristic (with the possible exception of travel direction, which we discuss further below). Since this seems to be emerging as a growing field of research, we hope in the future studies will be carried out that will permit greater differentiation of OD pairs. In the meantime, we adopt standardized profiles as a pragmatic approach that we believe yields realistic data useful for optimization benchmarks.

Finding the standardized demand profile. To determine the function $\mathbf{d}(t)$, our industry partner analysed the same shuttle route data as was used for deriving the fare profile (see Evans (2009) for more detail). In this case, the number of flights per hour was recorded across each of the two days investigated. The data for one of those days is plotted in Figure 3. Again, the data showed strong morning and evening peaks, with a soft midday peak. Figures 4 and 5 of Garrow et al. (2007) show similar results, with the morning and evening peaks occurring for one type of travellers, and the central peak for another. We thus again seek

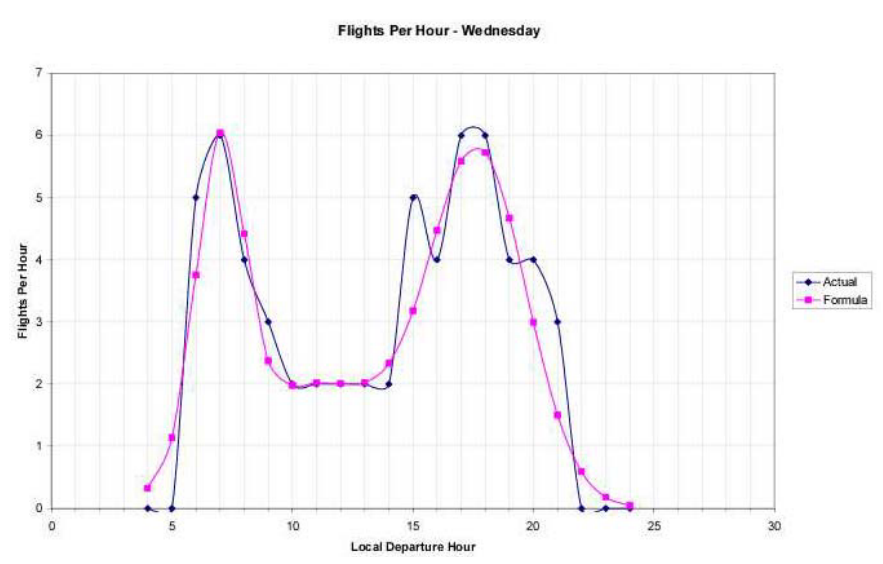

Figure 3: Actual and fitted demand profiles

\begin{tabular}{cccc}
\hline Type $(y)$ & $\mu_{y}$ & $\sigma_{y}$ & $w_{y}$ \\
\hline Morning & 7.0 & 1.0 & 5 \\
Midday & 11.0 & 3.5 & 2 \\
Evening & 17.75 & 2.0 & 5.5 \\
\hline
\end{tabular}

Table 2: Demand profile function parameters

to fit a combination of 3 normal distributions to this data, i.e. we construct

$$
\hat{\mathbf{d}}(t)=\beta^{a}(t)+\beta^{m}(t)+\beta^{e}(t)
$$

where each function took the form

$$
\beta^{y}(t)=w_{y} \exp \left(-\frac{\left(t-\tau_{y}\right)^{2}}{2 \sigma_{y}^{2}}\right)
$$

for each $y \in\{a, m, e\}$, where $\tau_{y}$ and $\sigma_{y}$ are the two normal distribution function parameters (representing mean and standard deviation respectively), and $w_{y}$ is a weighting factor. ( $\mathbf{d}(t)$ will be set to $\hat{\mathbf{d}}(t)$ multiplied by a normalizing factor.) The parameters found to best fit the two days of data are shown in Table 2, and the resulting function $\hat{\mathbf{d}}(t)$ is shown in Figure 3.

We note that Walker (2006) also suggests approximating the demand distribution by time of day with a combination of normal distributions.

Differentiating by direction of travel. As we have already mentioned, passengers' preferred travel time can be affected by the length of the trip, and its direction, in particular if a change in time zone is involved. Research presented by Walker (2006), Garrow et al. (2007) and Evans (2009) suggests that travel time preferences are similar for all directions of travel except for west-to-east, and the difference is more noticeable for travel which includes at least one time zone change, and takes more than 3 hours.

To illustrate, we give a sample of the results given in Evans (2009), obtained by analysing schedules operating at the end of 2007 for 44 carriers flying to 994 different airports in Europe, North America, Asia, Africa, South America and Australia, including all airlines in the Star alliance, all airlines in the oneworld alliance, Malaysia Airlines and Jetstar. The data 
used only included scheduled services, and did not include actual passenger data, so only the available capacity of each leg was used in the analysis. Plots of total flight capacity by local hour of departure are given for all non-west-to-east flights (with net west-east travel less than $750 \mathrm{~km}$ ), broken down by length of trip, in Figure 4. Similar plots for west-to-east travel are shown in Figure 5 (the case of trips less than $1000 \mathrm{~km}$ is omitted, because it is very similar to the non-west-to-east case).
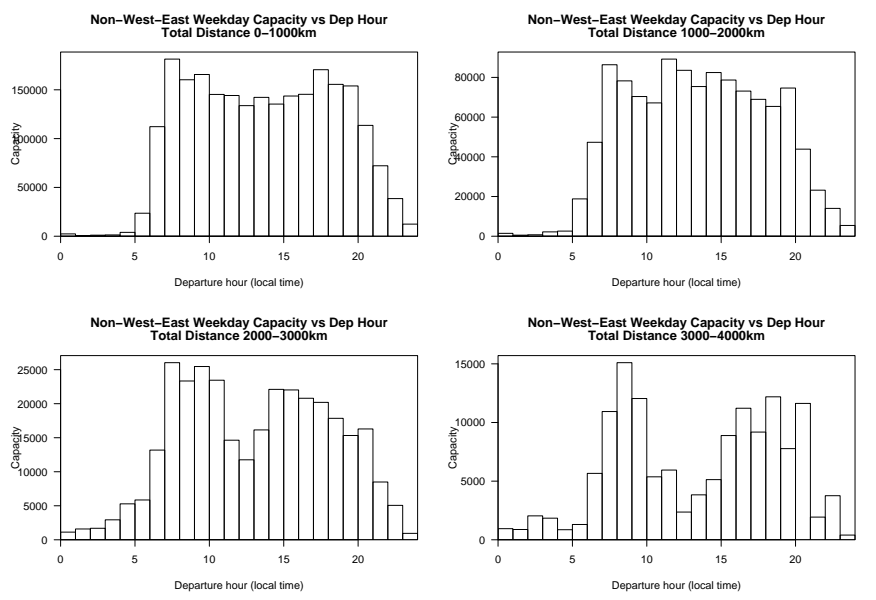

Figure 4: Non W-E Capacity versus local hour of departure

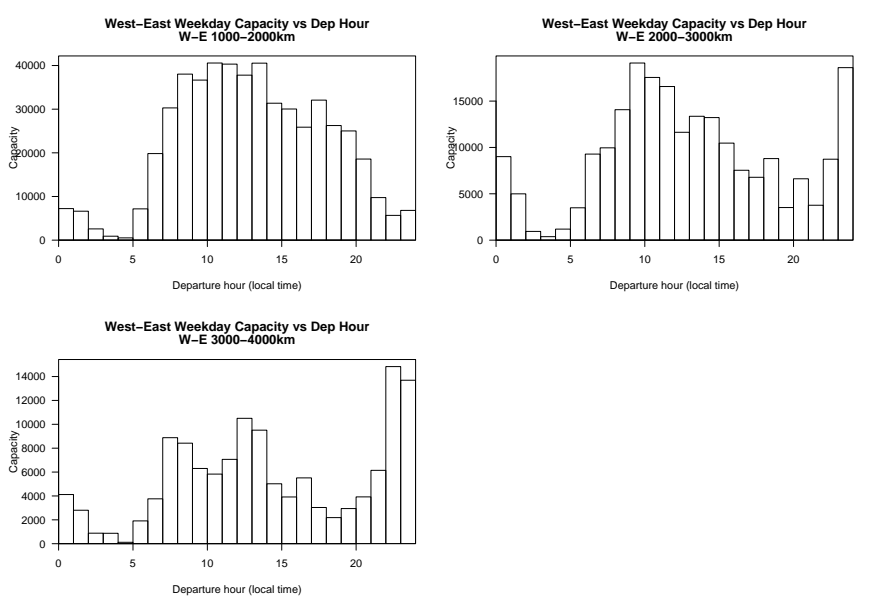

Figure 5: W-E Capacity versus local hour of departure

The differences are particularly apparent for trips of greater than $2000 \mathrm{~km}$. In these cases, it appears that a significant portion of the late afternoon/evening demand is shifted to arrive after $5 \mathrm{am}$ instead of before midnight. This may be a natural consequence of airport curfews, or may reflect actual passenger preferences.

Thus we adjust our demand profile function on OD pairs corresponding to long west-to-east trips, by shifting the portion of demand that would arrive between midnight and 5am to the next day. Mathematically, we construct an OD-specific demand profile

$\mathbf{d}_{o, d}(t)= \begin{cases}\mathbf{d}(t)+\mathbf{d}\left(T_{0}^{s}+t-T^{5 a m}\right), & T^{5 a m} \leq t \leq T^{5 a m}+T_{0}^{e}-T_{0}^{s} \\ 0, & T_{0}^{s} \leq t \leq T_{0}^{e} \\ \mathbf{d}(t), & \text { otherwise, }\end{cases}$

where $\left[T_{0}^{s}, T_{0}^{e}\right]$ is the interval of time during which trips departing $o$ can be expected to arrive at $d$ in the midnight to $5 \mathrm{am}$ interval, and $T^{5 a m}$ is obviously $5 \mathrm{am}$ in commensurate time units.

This is clearly something of an ad hoc adjustment, and in future work, we plan to derive fare and demand profiles, and adjust the timing for types of groups, differentiated by direction and length of travel.

\section{Benchmark problems}

In this section we present summary statistics of the 33 benchmark instances created using the methodology described in this paper, taking as input the flight network and OD-pair demand data generated in our first paper Akartunalı et al. (2009a). The complete set of instances and supporting material is available at the URL www . infotech.monash. edu . au/ wallace/airline benchmarks along with the reference Evans (2009).

The 33 instances consist of 30 with a single hub, and 3 with two hubs. The instances are grouped into threes. Each group of three networks were generated using the same parameters except the number of spokes which was varied to provide networks of different sizes. The instances show a variety of characteristics, from small to large in terms of the total passenger market, flying range, and size of the flight network. They also have differing degrees of asymmetry in the geometry of the flight network, as well as in the OD-pair demand, which also range over the proportion of passengers with an available direct service, versus those with at best a one-stop service. More details can be found in Akartunali et al. (2009a).

Here in Tables 3 and 4 we show for each instance the flight network size, total number of OD-pair demands, total number of passengers and summary statistics of the passenger load on arcs that was used to infer the OD-pair demand. We also give, in the column "Pax groups", the total number of passenger groups generated by the methodology we have described here, which ranges from just over 100 up to nearly 21,000 in the largest case. In the four columns headed "OD-pair pax groups" we give summary statistics of the number of passenger groups generated for each OD-pair. These range from 1, meaning that the expected frequency of service on that OD-pair was so low that only the time-insensitive group was generated, to a high of 29 . In the final four columns, headed "OD-pair max revenue" we give summary statistics for the total revenue available in each OD-pair market. This is calculated by multiplying the number of passengers in each group by its peak fare, and is the revenue that would be collected if every passenger could be carried at their preferred time of travel.

As can be seen from the tables, the instances display a range of features. They offer good opportunities for testing optimization approaches, with the ability to investigate performance against various characteristics, as well as to test scalability algorithms. 


\begin{tabular}{|c|c|c|c|c|c|c|c|c|c|c|c|c|c|c|c|c|c|}
\hline \multirow{2}{*}{ Instance } & \multicolumn{2}{|c|}{ Spoke } & \multirow{2}{*}{$\begin{array}{l}\text { OD } \\
\text { pairs }\end{array}$} & \multirow{2}{*}{$\begin{array}{l}\text { Pax } \\
\text { count }\end{array}$} & \multirow[b]{2}{*}{ avg } & \multicolumn{3}{|c|}{ Arc pax count } & \multirow{2}{*}{$\begin{array}{c}\text { Pax } \\
\text { groups }\end{array}$} & \multicolumn{4}{|c|}{ OD-pair pax groups } & \multicolumn{4}{|c|}{ OD-pair max revenue } \\
\hline & ports & Arcs & & & & stdev & $\min$ & $\max$ & & avg & stdev & $\min$ & $\max$ & avg & stdev & $\min$ & $\max$ \\
\hline H1A & 72 & 144 & 2496 & 72408 & 502.83 & 285.21 & 107 & 1472 & 2523 & 1.01 & 0.18 & 1 & 4 & 17288.8 & 72053 & 533.19 & 883862 \\
\hline $\mathrm{H} 2 \mathrm{~A}$ & 12 & 24 & 106 & 14160 & 590 & 327.26 & 212 & 1472 & 112 & 1.06 & 0.42 & 1 & 4 & 84056.4 & 143718 & 2415.36 & 920988 \\
\hline $\mathrm{H} 4 \mathrm{~A}$ & 72 & 144 & 2834 & 72408 & 502.83 & 285.21 & 107 & 1472 & 2861 & 1.01 & 0.17 & 1 & 4 & 15133.4 & 55983.2 & 556.61 & 769987 \\
\hline HAA & 24 & 48 & 450 & 71410 & 1487.71 & 1340.7 & 124 & 4860 & 1848 & 4.11 & 4.3 & 1 & 29 & 30682.3 & 89911 & 242.79 & 794261 \\
\hline HBA & 72 & 144 & 3374 & 106474 & 739.4 & 821.65 & 28 & 4860 & 6513 & 1.93 & 1.89 & 1 & 29 & 5808.23 & 30411.6 & 195.95 & 804883 \\
\hline HEA & 72 & 144 & 3393 & 106474 & 739.4 & 821.65 & 28 & 4860 & 6804 & 2.01 & 1.95 & 1 & 29 & 5749.71 & 29214.9 & 200.63 & 779450 \\
\hline HFA & 120 & 240 & 8955 & 224898 & 937.08 & 911.61 & 68 & 4860 & 20985 & 2.34 & 2.11 & 1 & 29 & 4424.3 & 23564.4 & 146.5 & 682306 \\
\hline HGA & 24 & 48 & 448 & 53230 & 1108.96 & 1189.57 & 83 & 4860 & 1412 & 3.15 & 3.64 & 1 & 29 & 25110.7 & 80359.1 & 247.47 & 895615 \\
\hline HHA & 72 & 144 & 3254 & 104278 & 724.15 & 808.63 & 28 & 4860 & 5930 & 1.82 & 1.81 & 1 & 29 & 6388.46 & 33215.8 & 219.37 & 775166 \\
\hline HIA & 120 & 240 & 8353 & 173132 & 721.38 & 789.65 & 36 & 4860 & 14877 & 1.78 & 1.73 & 1 & 29 & 4100.04 & 23395.4 & 200.63 & 665039 \\
\hline HJA & 24 & 48 & 468 & 53230 & 1108.96 & 1189.57 & 83 & 4860 & 1500 & 3.21 & 3.71 & 1 & 29 & 23719.6 & 65873.7 & 224.05 & 715108 \\
\hline HKA & 72 & 144 & 3333 & 104278 & 724.15 & 808.63 & 28 & 4860 & 6187 & 1.86 & 1.87 & 1 & 29 & 6170.27 & 27577.4 & 210 & 639773 \\
\hline HPA & 12 & 24 & 120 & 17564 & 731.83 & 810.76 & 50 & 2640 & 205 & 1.71 & 2.12 & 1 & 12 & 44741.6 & 111124 & 256.84 & 700961 \\
\hline HQA & 24 & 48 & 410 & 27824 & 579.67 & 643.89 & 45 & 2640 & 567 & 1.38 & 1.43 & 1 & 12 & 17270.9 & 48132.4 & 252.15 & 419344 \\
\hline HRA & 60 & 120 & 1821 & 51246 & 427.05 & 479.21 & 15 & 2640 & 2038 & 1.12 & 0.74 & 1 & 12 & 8520.44 & 46469.3 & 205.31 & $1.22 \mathrm{E}+006$ \\
\hline HSA & 12 & 24 & 127 & 20062 & 835.92 & 403.54 & 253 & 1472 & 175 & 1.38 & 1 & 1 & 4 & 67505.8 & 110607 & 828.28 & 620770 \\
\hline HTA & 24 & 48 & 435 & 23740 & 494.58 & 281.04 & 30 & 1472 & 441 & 1.01 & 0.21 & 1 & 4 & 26181.5 & 65973.8 & 369.25 & 542610 \\
\hline HUA & 60 & 120 & 2323 & 56192 & 468.27 & 256.74 & 155 & 1472 & 2329 & 1 & 0.09 & 1 & 4 & 11625.5 & 43499.3 & 359.89 & 584179 \\
\hline HVA & 12 & 24 & 136 & 20062 & 835.92 & 403.54 & 253 & 1472 & 196 & 1.44 & 1.07 & 1 & 4 & 62731.9 & 104070 & 903.23 & 530357 \\
\hline HWA & 24 & 48 & 441 & 23740 & 494.58 & 281.04 & 30 & 1472 & 447 & 1.01 & 0.21 & 1 & 4 & 25760.3 & 63262 & 472.3 & 495093 \\
\hline HXA & 60 & 120 & 2466 & 56192 & 468.27 & 256.74 & 155 & 1472 & 2472 & 1 & 0.09 & 1 & 4 & 10907.3 & 39692.7 & 355.2 & 546277 \\
\hline HYA & 12 & 24 & 100 & 14160 & 590 & 327.26 & 212 & 1472 & 106 & 1.06 & 0.43 & 1 & 4 & 89404.3 & 176875 & 907.91 & $1.04 \mathrm{E}+006$ \\
\hline HZA & 24 & 48 & 298 & 23232 & 484 & 290.63 & 30 & 1472 & 304 & 1.02 & 0.25 & 1 & 4 & 47093.1 & 115725 & 832.97 & 804993 \\
\hline
\end{tabular}

Table 3: Summary statistics for single hub benchmark instances

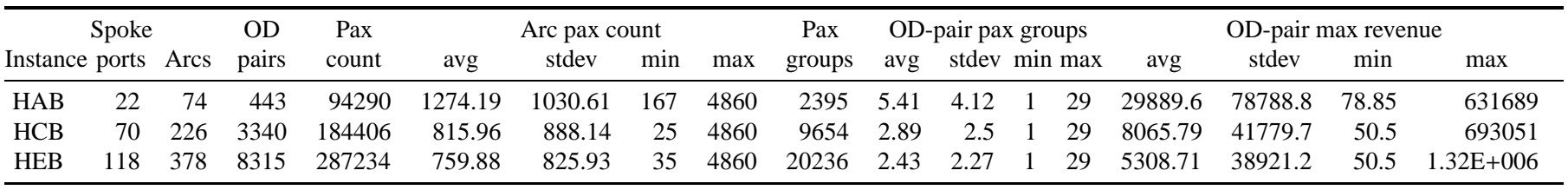

Table 4: Summary statistics for two hub benchmark instances 


\section{Conclusions and future work}

In this paper, we have argued that market segmentation and the use of passenger groups represent a natural "next step" in passenger choice models for use in airline planning optimization. We have described one approach to defining such groups, which attempts to balance sometimes divergent empirical insights against what can practically be instantiated using currently available data. We have presented a methodology by which realistic passenger group data can be generated from a given flight network and OD-pair demands, and produced 33 benchmark instances exhibiting a range of characteristics useful for testing optimization algorithms.

The data we have presented here can be used immediately for testing alternative passenger choice models, provided an airline schedule is also available. We intend in future work to generate schedules to accompany this data, indeed Akartunalı et al. (2009b) explores first steps in this direction. Clearly the generation of realistic schedules depends on the available airline resources. While much data on aircraft types and configurations, airline fleets, and operating costs is publicly available, in future work, we plan to extend our benchmark data to include aircraft, and so provide complete sets of input data for the flight schedule design process. We hope that in doing so we can stimulate further research in this hitherto less-studied area of airline planning.

\section{Acknowledgements}

The authors are very grateful to Ian Evans and Alan Dormer (CTI Pty Ltd) for their ongoing support and guidance on a variety of practical airline-related issues and for numerous technical suggestions and insightful feedback that improved the content and exposition of this work. This research is supported by the Australian Research Council, under Linkage Projects LP00668076 and LP0883855, and by CTI Pty Ltd.

Akartunalı, K., Boland, N., Evans, I., Wallace, M., Waterer, H., 2009a. Airline planning benchmark problems. Part I: Characterizing networks and demand with limited data. Tech. rep., University of Newcastle.

Akartunalı, K., Boland, N., Evans, I., Wallace, M., Waterer, H., Smith, O., 2009b. Airline schedule design: Network design optimization and heuristics. Working paper, The University of Melbourne.

Bazargan, M., 2004. Airline Operations and Scheduling. Ashgate Publishing Ltd.

Civil Aviation Authority, 2008. CAA passenger survey report. Tech. rep., Economic Regulation Group.

Coldren, G., Koppelman, F., Kasturirangan, K., Mukherjee, A., 2003. Modeling aggregate air-travel itinerary shares: logit model development at a major US airline. Journal of Air Transport Management 9, 361-369.

Commission of the European Communities, 2007. Ryanair/Aer Lingus merger procedure report. Office Journal of the European Union Case No COMP/M.4439.

Evans, I., 2009. Typical airline demand and fare profiles. Tech. rep., Constraint Technologies International.

Garrow, L., Jones, S., Parker, R., 2007. How much airline customers are willing to pay: an analysis of price sensitivity in online distribution channels. Journal of Revenue and Pricing Management 5, 271-290.

Klabjan, D., 2005. Large-scale models in the airline industry. In: Desaulniers, G., Desrosiers, J., Solomon, M. M. (Eds.), Column Generation. Springer, pp. 163-196.

Kniker, T. S., 1998. Itinerary-based airline fleet assignment. Ph.D. thesis, Massachusetts Institute of Technology.
Koppelman, F., Coldren, G., Parker, R., 2008. Schedule delay impacts on airtravel itinerary demand. Transportation Research Part B 42, 263-273.

Lohatepanont, M., Barnhart, C., 2004. Airline schedule planning: Integrated models and algorithms for schedule design and fleet assignment. Transportation Science 38 (1), 19-32.

UK Department of Transport, 2006. National travel survey: 2006. Tech. rep., http://www.dft.gov.uk/adobepdf/162469/221412/221531/223955/322743/NTS2006V3.pdf.

Walker, J., 2006. Time of day in airline passenger demand, presentation at the INFORMS Annual Meeting, Pittsburgh PA.

Yan, S., Tang, C.-H., Lee, M.-C., 2007. Aflight scheduling model fortaiwan airlines under market competitions. Omega 35 (1), 61-74.

Yan, S., Tseng, C.-H., 2002. A passenger demand model for airline flight scheduling and fleet routing. Computers and Operations Research 29, 15591581 . 